\title{
Surveys of organic gunshot residue prevalence: comparison between civilian and police populations
}

\author{
Manuela Manganelli ${ }^{\mathrm{a}, \mathrm{b}}$, Céline Weyermann ${ }^{\mathrm{a}}$ and Anne-Laure Gassner ${ }^{\mathrm{a}}$
}

\author{
${ }^{a}$ Ecole des Sciences Criminelles, University of Lausanne, Batochime, 1015 Lausanne, \\ Switzerland \\ ${ }^{\mathrm{b}}$ Kantonspolizei Aargau, Polizeikommando, 5000 Aarau, Switzerland
}

\section{Abstract}

This study investigated the prevalence of eight OGSR compounds in a civilian and a police population. Specimens were collected from the hands and sleeves of 122 civilians and 115 individuals working in police services using carbon stubs. Data was acquired using liquid chromatography coupled to tandem mass spectrometry.

Results indicated a non-negligible prevalence in the civilian sample, with $18 \%$ of the 122 civilians sampled having one or more OGSR related compounds on their hands and $11.5 \%$ on their wrists or sleeves. For the police population, the prevalence was logically higher than for civilians, with $36.5 \%$ of the hand specimens and 33\% of the wrist specimens positive for one or more compounds. A higher prevalence was expected for the second population due to the possession of service weapons regularly used during shooting exercises. These results demonstrate that the presence of one OGSR compound is not a rare occurrence, even in a civilian population. Considering the results of this research together with information on alternative sources of the targeted OGSR compounds can serve as a basis for OGSR interpretation in casework.

Keywords: Forensic science, firearms, firearm discharge residue, contamination 


\section{INTRODUCTION}

According to the Small Arms Survey, the estimated number of firearms all over the world, licit and illicit, might exceed one billion [1]. They are divided between firearms held by civilians (84.6\%), state militaries (13.1\%) and law enforcement agencies (2.2\%). There proportions are highly countrydependent due to various policies on firearms, crime rates and ongoing armed conflicts. Incidents involving firearms are usually investigated by forensic science services. Gunshot residues (GSR) produced during a firearm discharge can be detected and analysed in casework to evaluate if a person has been involved in a shooting incident [2]. During a discharge, GSR are ejected from all firearm openings and deposited onto the shooter and various surfaces in close proximity. They originate from the primer and propellant, as well as other firearm and ammunition components such as metals (from the bullet, cartridge case or gun barrel) and lubricant [3, 4]. GSR are classified into primer residues, also called inorganic GSR (IGSR), and propellant residues that will be referred to as organic GSR (OGSR) [2]. It is important to note that even though IGSR are called primer residues, their chemical composition is not limited to that of the primer, but contributions from other metallic surfaces involved in the discharge or to bluing products used to protect firearms from corrosion [5] have been observed. The analysis of IGSR is routinely performed by scanning electron microscopy coupled to energy dispersive X-ray spectroscopy (SEM-EDX) [2], whereas OGSR usually remain unexploited in routine analyses.

GSR can be transferred primarily to the shooter, but also to their clothing, a bystander, the victim or any object or surface in close proximity. The amount of OGSR transferred will depend on the ammunition (composition, combustion efficiency) and firearm (model, memory effect from previous ammunition, lubricant) used. The number of cartridges shot might also have an influence. Moreover, GSR might be further transferred to other surfaces and individuals through processes called secondary transfer and tertiary transfer. Thus, it might be possible to detect GSR on an individual not involved in any firearm discharge. As indicated in the literature, such events are not negligible and should be considered when interpreting GSR traces [6-10]. Therefore, it is essential to estimate the chances of an individual testing positive for GSR without being involved in a firearm incident.

To date, the prevalence of IGSR has been investigated in various populations. Lucas et al. studied the occurrence of IGSR in a random population of 289 Australians [11]. Their results showed an overall prevalence of $0.3 \%$ for characteristic $\mathrm{PbBaSb}$ particles, $8 \%$ for $\mathrm{PbSb}$ and about $7 \%$ for single $\mathrm{Pb}, \mathrm{Ba}$ or Sb particles. In Poland, Brozek-Mucha studied a population of 100 non-shooters and 50 shooters [9]. Only one PbBaSb particle was detected among individuals who had no contact with firearms, whereas the numerous particles found among shooters showed a strong correlation with the time elapsed since the last shooting session. The prevalence of IGSR was also investigated among police officers. Gialamas et al. found 3 specimens with one $\mathrm{PbBaSb}$ particle in a population of 43 nonshooting police officers in California [12]. In Canada, Gerard et al. detected at least one $\mathrm{PbBaSb}$ particle on the hands of $60 \%$ of patrol and plainclothes officers (66 individuals) and on $24 \%$ of their 
equipment [13]. In the same study, no IGSR particles were found on the 28 civilians working in a police environment and only 2 of the 18 vehicles sampled had one characteristic GSR particle. In Australia, Cook sampled 33 police officers immediately after the start-of-shift handling of their firearm and $28 \mathrm{had} \mathrm{PbBaSb}$ particles on their hands, with an average of 64 such particles [14]. Another study by Lindsay et al. targeted 13 employees of firearm manufactures [15]. $\mathrm{PbBaSb}$ particles were found on nine of the employees, and no more than two characteristic particles were found on the hands of the five individuals who had no direct contact with firearms or items that had been in the firing range. For the other four employees, the number of particles ranged from nine to 424. In the United States, Berk et al. investigated police vehicles and detention facilities in Chicago [16]. A total of 201 specimens were analysed and a total of $56 \mathrm{PbBaSb}$ particles were found in 23 specimens. Only two vehicles had one particle. The other particles were recovered from detention facilities with a maximum of seven particles collected from a table surface and restraining bars. Finally, in Ireland, Hannigan et al. collected 100 upper body garments submitted to a forensic laboratory that were not related to a firearm offence [17]. 98\% of the specimens collected from the cuffs were negative: up to two $\mathrm{PbBaSb}$ particles were detected on two garments. The aforementioned studies showed that prevalence can considerably differ depending on the targeted population, people and objects. The items/people directly in contact with firearms generally presented the largest prevalence.

In the case of IGSR, the elemental composition, the morphology and the composition of the entire particle population of the specimen are considered by the analyst [18]. However, due to the common use of bulk analytical techniques such as micellar electrokinetic capillary electrophoresis (MEKC) [19-22], gas chromatography (GC) [23-25] or liquid chromatography coupled to mass spectrometry (LC-MS) [26-30], the sole overall specimen composition can be obtained for OGSR. A positive result is based on the detection of an OGSR compound, regardless of its source. Hence, the knowledge of potential alternative sources of OGSR compounds is a prerequisite for the interpretation of that trace. Currently, there is only limited or obsolete information regarding alternative sources, leading to a lack of precise definition of which OGSR compounds are the most characteristic [31]. However, if a sufficient amount of data is collected, prevalence studies might also help in identifying alternative sources. Few prevalence researches have been published concerning OGSR. Various explosive background studies including the detection of nitroglycerine (NG) and dinitrotoluenes (DNT), which are also major components of smokeless powders, indicate a low prevalence of these compounds in public places [32-34]. Regarding OGSR prevalence, the hands of 100 volunteers were sampled in the United States and no specimen tested positive using MEKC [19]. Another study based on a sample of 73 people including law enforcement personnel of Morgantown, West Virginia (USA), showed less than $5 \%$ of positive results for the specimens analysed by ion-mobility spectrometry (IMS) [35]. The authors used neural networks and the results were based on pattern matching. Thus, the proportion of positive results depended on the threshold defined by the user. In that study, when a likelihood ratio of 
10:1 was used, the frequency of positives dropped under $2 \%$. In Switzerland, a preliminary study concerning 27 people detected no positive specimen using LC-MS and also concluded that OGSR occurrence was rare [36]. Furthermore, as suspects might be contaminated during an arrest, transportation in a police car or detention, it is important to estimate the level of contamination of police officers and premises. Hofstetter et al. collected specimens from 25 individuals working in a forensic laboratory [36], only two positive results were observed. Ali et al. [37] collected seventy specimens from four Pittsburgh police stations and vehicles and analysed both IGSR and OGSR. Only one characteristic IGSR particle was detected, whereas ethylcentralite (EC) was the only organic compound quantified in four specimens. Altogether, even though the aforementioned studies indicate a rather low prevalence, the amount of prevalence data for OGSR molecules required to interpret that trace evidence remains very limited. More studies using sensitive instrumental techniques and a higher number of specimens need to be carried out.

In order to gain more knowledge about the prevalence of OGSR in Switzerland, the present work investigated the detection of eight OGSR compounds in two populations to provide useful information for the assessment of OGSR evidence. Specimens were collected from 122 civilians and 115 individuals working in a police environment. Collection was performed using carbon stubs and data was acquired using LC-MS/MS with electrospray ionisation in positive mode. The results obtained from both populations were compared and discussed in a forensic perspective.

\section{MATERIAL AND METHODS}

\subsection{Target populations}

Specimens were collected from two populations. The first one is a so called civilian population composed of 122 volunteers with no or limited contact with firearms. All of them completed a questionnaire evaluating their firearm exposure. Data such as occupation, gender, hunting/military activity and firearm ownership (model) were collected. If volunteers indicated ownership or use of a firearm, data regarding the ammunition type, the frequency of use, the date of the last shooting session and the number of cartridges discharged was also collected. Finally, they were also asked about the last time they washed their hands and whether they thought there was a reason they would test positive for OGSR.

The second survey targeted a police population. This included police officers, police recruits as well as scientific collaborators working in three regional Swiss police services. 115 individuals were sampled and filled in a questionnaire similar to the one submitted to the civilian population, with an additional question included regarding the use of a firearm outside the purpose of their profession.

\subsection{Specimen collection and preparation}


Specimens were collected using carbon stubs from Plano (Wetzlar, Germany), consisting of an adhesive carbon tab $12 \mathrm{~mm}$ in diameter mounted on a $12.5 \mathrm{~mm}$ aluminium stub. This assembly was inserted in a plastic vial with a screwed cap. Two stubs were used for each participant, one for their hands and the other for their wrists or the cuffs of their sleeves (depending whether they wore a short or long-sleeved shirt). Following recommendations from Zeichner et al. [38], the stubs were dabbed about 100 times on the skin and 200 times on the sleeves. For the hands, sample collection was first performed on the thumb-index region and then on the back and palm. To minimize contaminations, the person in charge of specimen collection wore gloves and changed them after each participant's sampling. It must be noted that 50 specimens (civilian population) were auto-collected, that is to say that the participants were given a sampling kit to stub themselves. Prior to that, a presentation explaining the goal of the study and the sampling procedure was given.

To extract the compounds present in the specimens, the carbon adhesive was removed from the stub with clean tweezers and transferred to a $20 \mathrm{~mL}$ scintillation vial containing $1 \mathrm{~mL} \mathrm{MeOH}$. After 15 minutes ultrasonication at room temperature, the resulting solution was filtered through a $0.2 \mu \mathrm{m}$ Chromafil PTFE syringe filter (Macherey-Nagel, Düren, Germany) to remove carbon particles. To detect potential laboratory contamination during specimen preparation, methanol blanks were prepared before and after each extraction session. Likewise, a blank carbon tab was extracted to check for potential contamination of the stub batch. For all these control samples, no OGSR were detected.

\subsection{Chemicals}

Acetonitrile, methanol, formic acid (FA) and water were of ULC-MS grade (Sigma-Aldrich, Buchs, Switzerland). The study targeted eight OGSR compounds: diphenylamine (DPA) from Fluka (Buchs, Switzerland); ethylcentralite (EC), $N$-nitrosodiphenylamine ( $N$-nDPA), 4-nitrodiphenylamine (4nDPA), akardite II (AK II) and N,N-diphenylformamide ( $N, N$-DPF) from Sigma-Aldrich (Buchs, Switzerland); 2-nitrodiphenylamine (2-nDPA) from Alfa Aesar (Karlsruhe, Germany); methylcentralite (MC) from MP Biomedicals (Illkirch, France). Standard solutions at $1 \mathrm{mg} / \mathrm{mL}$ were prepared in $\mathrm{MeOH}$ and stored at $4^{\circ} \mathrm{C}$.

\subsection{Instrumentation}

The specimens were analysed using an Agilent Infinity 1290 ultra-high performance liquid chromatography (UHPLC) from Agilent Technologies. The instrument was equipped with a binary pump enabling a maximum delivery flow rate of $5 \mathrm{~mL} / \mathrm{min}$, an autosampler, and a thermostatically controlled column compartment. Separation was performed using a C18 Kinetex core-shell column from Phenomenex $(2.6 \mu \mathrm{m}, 2.1 \mathrm{~mm} \times 100 \mathrm{~mm})$. A SecurityGuard ULTRA cartridge with C18 selectivity was used to protect the analytical column. The UHPLC system was coupled to a triple quadrupole mass spectrometer (5500 QTrap) from AB Sciex. Electrospray ionization was operated in 
positive mode. $[\mathrm{M}+\mathrm{H}]^{+}$was defined as the precursor ion for each of the target compounds, and quantification was obtained from the SRM measurements. The source parameters were as follows: the desolvation temperature was set to $500^{\circ} \mathrm{C}$, the nebulizer gas to $60 \mathrm{psig}$, the turbo gas to $50 \mathrm{psig}$ and the curtain gas to $25 \mathrm{psig}$. The IonSpray voltage was adjusted to $5500 \mathrm{~V}$. Data acquisition, treatment and instrument control were monitored using Analyst software. Details of the LC method, MS/MS parameters and limits of detection (LOD) can be found in Tables 1 and 2.

Table 1: LC parameters [30]

\begin{tabular}{|c|c|c|c|}
\hline Column type & \multicolumn{3}{|c|}{$\mathrm{C} 18(2.6 \mu \mathrm{m}, 2.1 \mathrm{~mm} \times 100 \mathrm{~mm})$} \\
\hline Column temperature & \multicolumn{3}{|l|}{$40^{\circ} \mathrm{C}$} \\
\hline Flow rate & \multicolumn{3}{|c|}{$0.25 \mathrm{~mL} / \mathrm{min}$} \\
\hline Injection volume & \multicolumn{3}{|l|}{$5 \mu \mathrm{L}$} \\
\hline \multirow[t]{5}{*}{ Gradient table } & $\mathrm{t} / \mathrm{min}$ & $\begin{array}{l}\% \mathrm{~A} \\
\mathrm{H}_{2} \mathrm{O}+0.1 \% \mathrm{FA}\end{array}$ & $\begin{array}{l}\% \mathrm{~B} \\
\mathrm{ACN}+0.1 \% \mathrm{FA}\end{array}$ \\
\hline & 0 & 65 & 35 \\
\hline & 0.5 & 65 & 35 \\
\hline & 6 & 20 & 80 \\
\hline & 7 & 0 & 100 \\
\hline
\end{tabular}

Table 2: MS parameters [30]

\begin{tabular}{|c|c|c|c|c|}
\hline & $\begin{array}{c}\text { SRM } \\
\text { transitions }\end{array}$ & $\begin{array}{c}\text { LOD } \\
{[\mathrm{ng} / \mathrm{mL}]}\end{array}$ & $\begin{array}{l}\text { Declustering } \\
\text { potential } \\
\text { [V] }\end{array}$ & $\begin{array}{c}\text { Collision } \\
\text { energy } \\
\text { [V] }\end{array}$ \\
\hline $\begin{array}{l}\text { Akardite II } \\
\text { (AK II) }\end{array}$ & $\begin{aligned} 227.1 & \rightarrow 170.1 \\
227.1 & \rightarrow 91.9\end{aligned}$ & 0.01 & 120 & $\begin{array}{l}27 \\
36\end{array}$ \\
\hline $\begin{array}{l}\text { Methylcentralite } \\
\text { (MC) }\end{array}$ & $\begin{array}{l}241.2 \rightarrow 134.1 \\
241.2 \rightarrow 105.9\end{array}$ & 0.01 & 125 & $\begin{array}{l}24 \\
36\end{array}$ \\
\hline $\begin{array}{c}N, N- \\
\text { diphenylformamide } \\
(N, N-\mathrm{DPF})\end{array}$ & $\begin{array}{l}198.1 \rightarrow 92 \\
198.1 \rightarrow 65\end{array}$ & 0.02 & 130 & $\begin{array}{l}30 \\
54\end{array}$ \\
\hline $\begin{array}{l}\text { Ethylcentralite } \\
\text { (EC) }\end{array}$ & $\begin{array}{c}269.2 \rightarrow 147.9 \\
269.2 \rightarrow 120\end{array}$ & 0.01 & 120 & $\begin{array}{l}20 \\
33\end{array}$ \\
\hline $\begin{array}{l}\text { 2-nitrodiphenylamine } \\
\text { (2-nDPA) }\end{array}$ & $\begin{array}{c}215.1 \rightarrow 197 \\
215.1 \rightarrow 180.1\end{array}$ & 0.02 & 80 & $\begin{array}{l}14 \\
23\end{array}$ \\
\hline $\begin{array}{l}\text { 4-nitrodiphenylamine } \\
\text { (4-nDPA) }\end{array}$ & $\begin{array}{l}215.1 \rightarrow 197.8 \\
215.1 \rightarrow 167.1\end{array}$ & 0.02 & 60 & $\begin{array}{l}18 \\
47\end{array}$ \\
\hline $\begin{array}{l}\text { Diphenylamine } \\
\text { (DPA) }\end{array}$ & $\begin{array}{l}170.1 \rightarrow 93 \\
170.1 \rightarrow 66\end{array}$ & 0.5 & 200 & $\begin{array}{l}32 \\
58\end{array}$ \\
\hline $\begin{array}{c}N- \\
\text { nitrosodiphenylamine } \\
(N-\text {-nitrosoDPA })\end{array}$ & $\begin{aligned} 199.1 & \rightarrow 169 \\
199.1 & \rightarrow 66\end{aligned}$ & 0.02 & 60 & $\begin{array}{l}15 \\
30\end{array}$ \\
\hline
\end{tabular}

186 Semi-quantitative data were obtained from a calibration curve (11 levels, 2 replicates) measured for each sequence of experiments with levels ranging between the LOD and $10 \mathrm{ng} / \mathrm{ml}$, except for DPA with a $100 \mathrm{ng} / \mathrm{mL}$ highest level. 


\section{RESULTS AND DISCUSSION}

\section{1}

192

193

194

195

196

197

198

199

200

201

202

203

204

205

206

207

208

209

\subsection{Civilian population}

The civilian population consisted of 122 participants. The questionnaires were used to extract information relative to the occupations of the participants and to evaluate their potential exposure to GSR in order to help interpret positive results. Among the 122 individuals, the gender was equally distributed with 61 men and 61 women. 68 students were sampled, representing 55.7\% of the whole dataset. The majority of the participants (101 people) did not possess a firearm (Fig. 1a), while 11 had a military rifle, 4 owned a pistol ( $9 \mathrm{~mm}$ calibre), 4 owned more than one firearm. Two people did not answer that question. The frequency of firearm use (Fig. 1b) was divided into three categories: "never", "rarely" (people who shoot at least once a year) and "regularly" (those who shoot at least once a month). It was observed that less than $5 \%$ of the individuals regularly used firearms. Only one individual reported discharging a firearm in the two weeks before specimen collection. It must be emphasized that this participant was sampled only ten minutes after the last shooting practice, did not wash his hands after shooting, and as expected, tested positive for OGSR. Of the $82.8 \%$ of the individuals who did not own a firearm, $73.8 \%$ of them reported having never shot. Finally, the participants were asked if they thought they might be contaminated. Seven individuals answered positively. Their explanations for potential contamination were the following: cleaning, manipulating or discharging a firearm (four participants), visiting premises contaminated with GSR (two participants) and installing a stand in the boot of a police vehicle (one participant).

a) Firearm possession

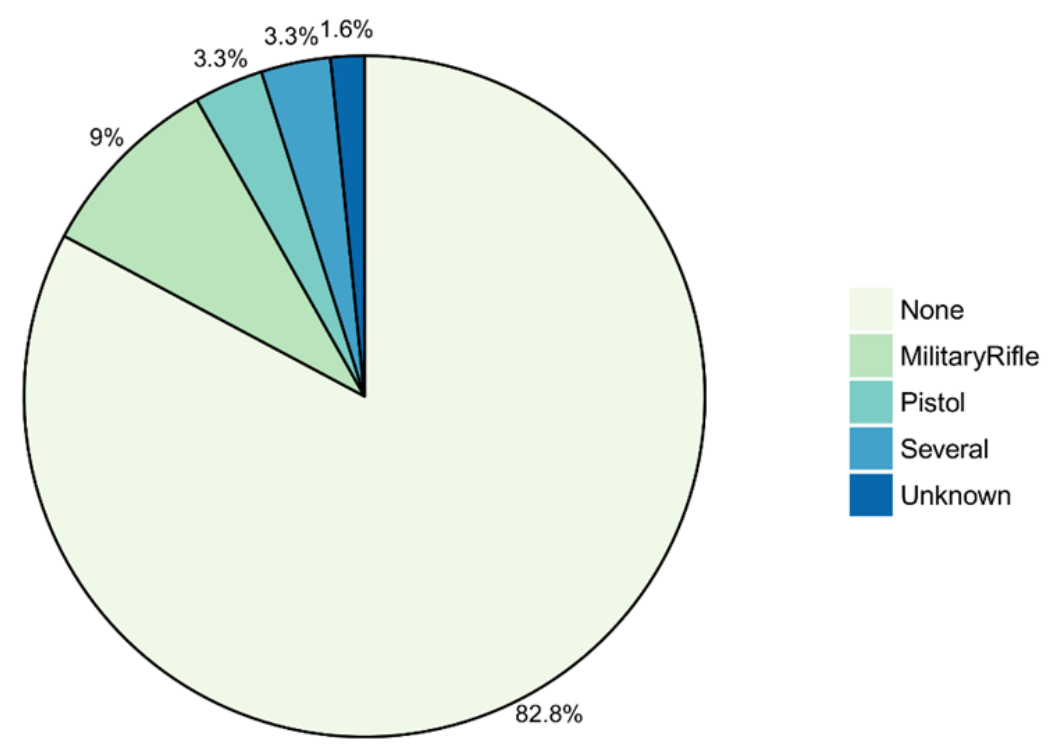


b) Firearm use frequency

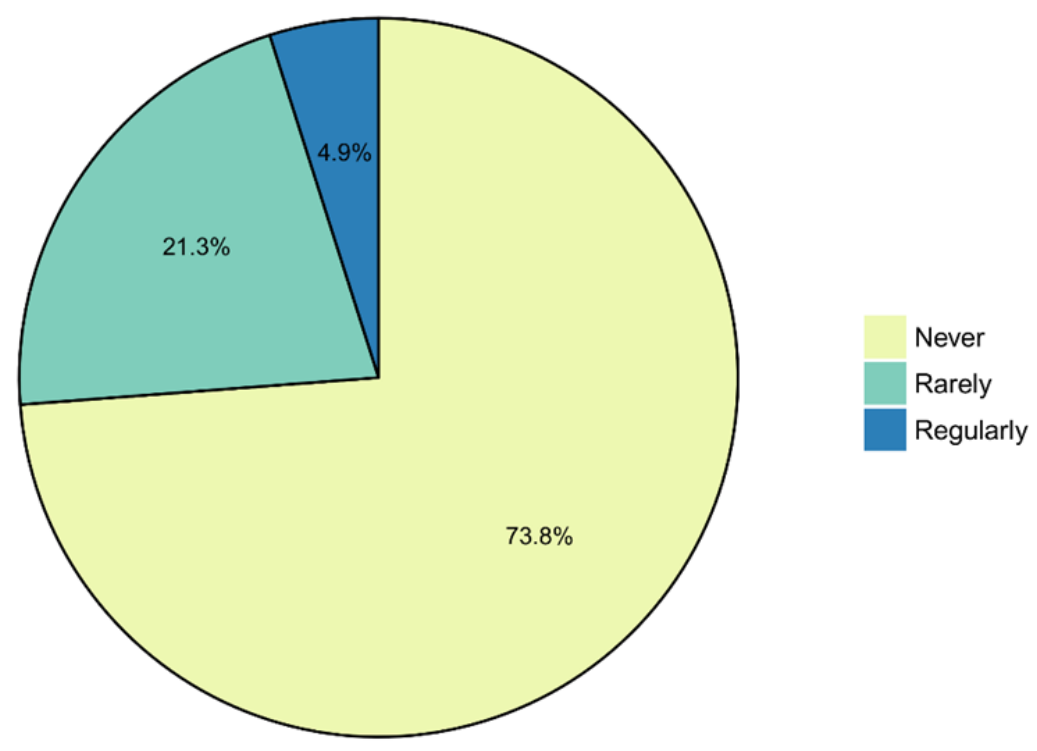

Figure 1: a) Firearm possession and b) Frequency of firearm use in the population of 122 civilians

214 Among the 122 individuals sampled in this study, only a minority tested positive for OGSR (Fig. 2a).

215 For the hand specimens, 100 individuals (82\%) were free from OGSR, 18 individuals (14.8\%) had one

216 OGSR compound, three individuals (2.5\%) had two compounds and only one individual had six

217 compounds on their hands. Comparable results were found for the sleeve specimens with slightly

218 fewer participants testing positive. In fact, 108 individuals (88.5\%) showed no OGSR, while 12 people

219 (9.8\%) had one compound, and two people had two and four compounds respectively. It is interesting

220 to note that the hand and sleeve specimens with the highest number of compounds were not collected

221 from the same person. When considering the hands and sleeves together, a higher percentage of people

222 were contaminated with at least one compound (Fig. 2a, green bars). Thus, it might be interesting to

223 collect specimens from other surfaces than hands as hands are generally washed much more frequently

224 than the face and hair, for example [36, 39]). 


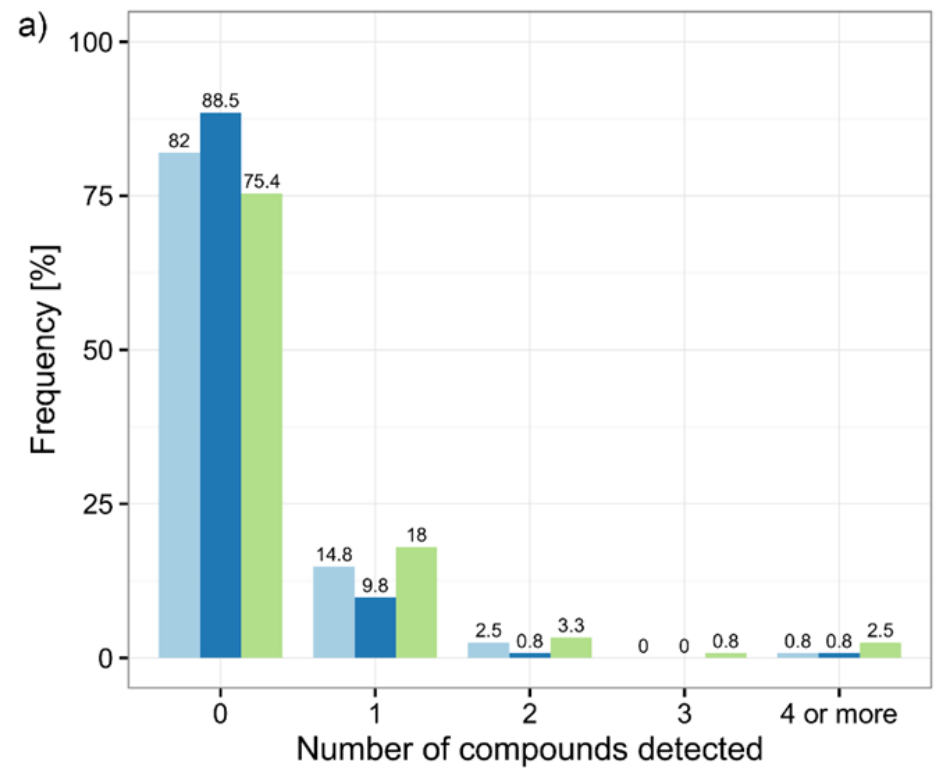

\section{Target

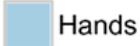 \\ Sleeves \\ Combined}

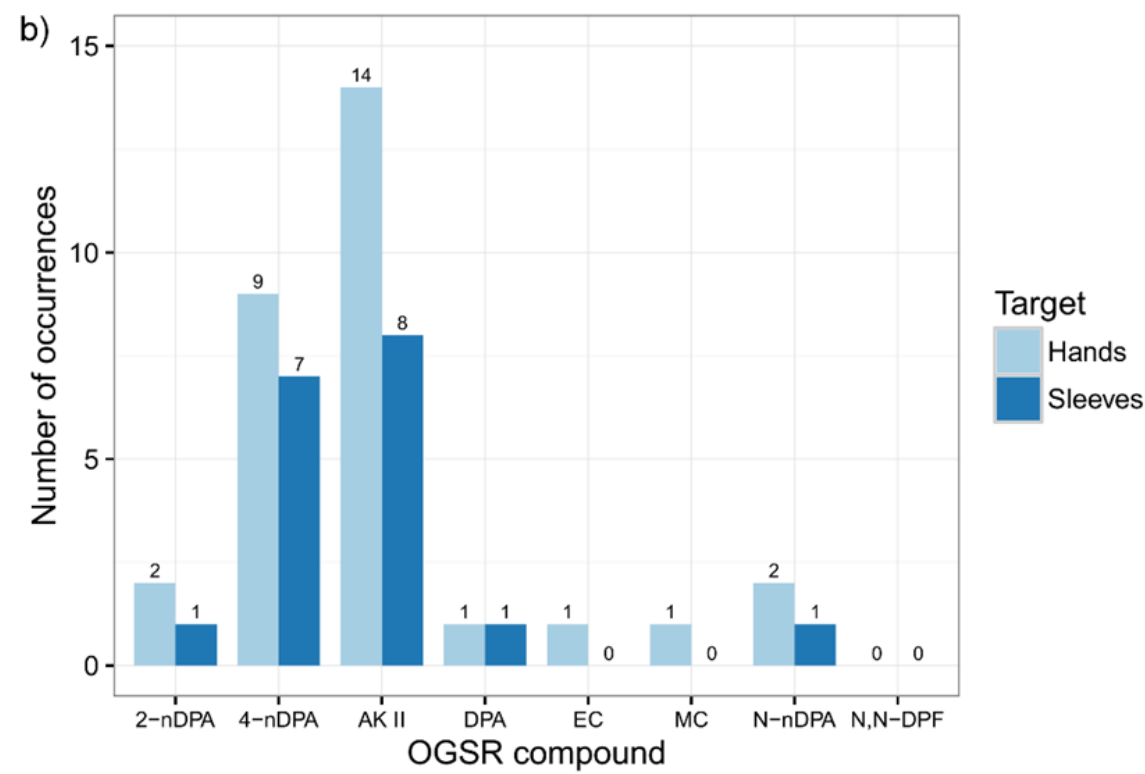

Figure 2: Prevalence in the civilian population: a) Prevalence versus number of compounds detected. The "combined" bar considers the total number of compounds detected on both hands and sleeves irrespective of potential compound duplication. For example, if DPA was detected on the hands and the same compound was detected on the sleeves, two compounds were counted. b) Number of positive specimens for each analyte.

Regarding the number of positive results per compound (Fig. 2b), it can be seen that AK II ( $47 \%$ and $44 \%$ of positive results for hands and sleeves respectively) and 4-nDPA (30\% and 39\% of positive results) were the most frequently detected compounds. Other DPA derivatives and centralites were rarely detected and $N, N$-DPF was never detected. The amounts detected (Fig. 3) were in the low ng range and very often close to the LOD. Such amounts are in the same range as those observed in persistence and secondary transfer studies [10,40], showing that it might be very difficult to distinguish between firearm discharge and incidental contamination. 


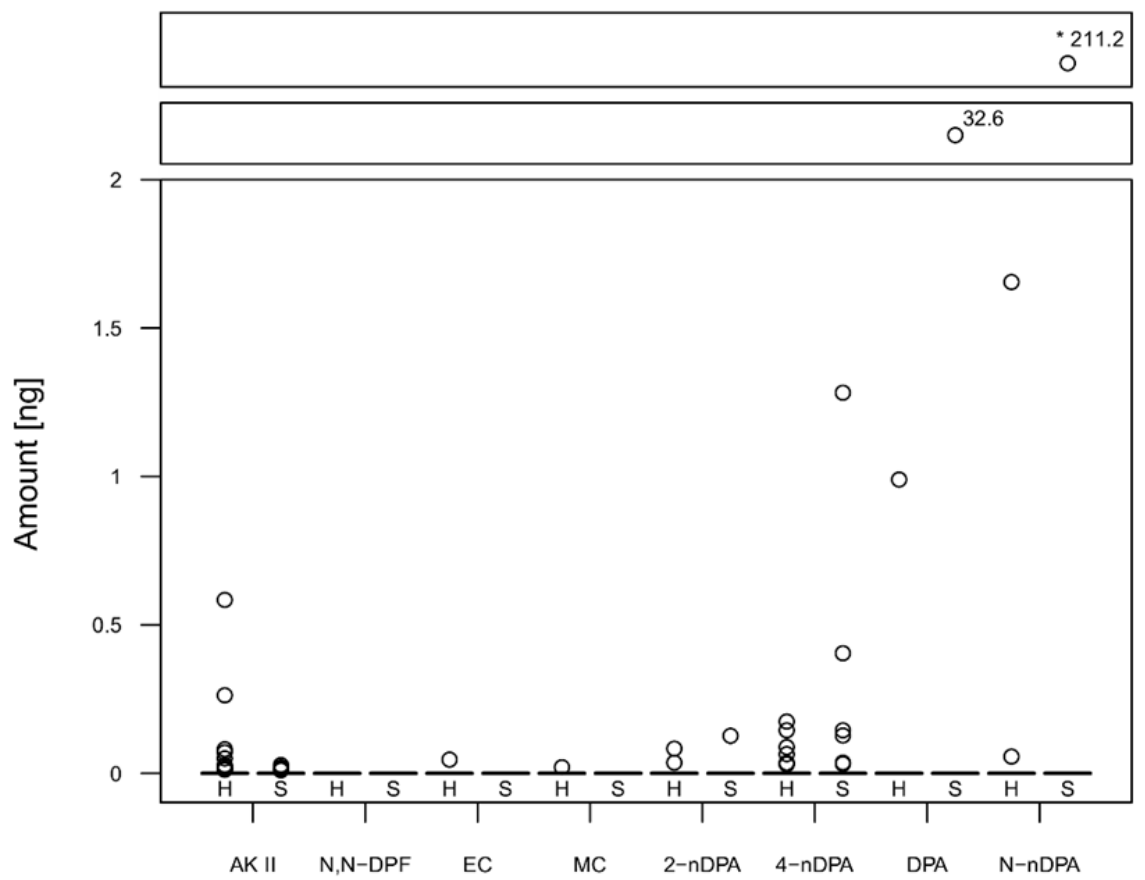

Figure 3: Prevalence in the civilian population: Amount of analyte detected. The asterisk indicates an extrapolated value (outside of calibration range)

The data from the questionnaire were investigated to look for a potential explanation for the presence of OGSR compounds. Two individuals had four or more compounds detected on their hands and sleeves respectively. The person who had six compounds on his hands (AK II, EC, DPA, N-nDPA, 4nDPA and 2-nDPA) was the participant (student) who discharged a firearm less than ten minutes before specimen collection without washing his hands and thus expected testing positive for OGSR. Interestingly, only AK II was detected on his sleeves. The person who had four compounds on his sleeves (DPA, $N$-nDPA, 4-nDPA and 2-nDPA) and no compounds detected on his hands was a student who never discharged a firearm. In that case, the presence of these compounds could not be explained by the questionnaire and might be due to contamination through secondary transfer. Three compounds were only detected when the results of the hands and sleeves were added (Fig. 2a). However, three individuals tested positive for two compounds, one only on the hands (4-nDPA and $N$-nDPA), one with two compounds on the hands and one on the sleeves (AK II, 4-nDPA and 4-nDPA respectively) and one with the same two compounds on the hands and sleeves (AK II and 4-nDPA). These three individuals had not manipulated or discharged a firearm recently. One of them (AK II and 4-nDPA on both hands and sleeves) thought a positive result was possible because he had installed a stand in the boot of a police vehicle. That person also owned a military rifle that he had not used for more than ten months. In that case, an explanation might be the contact with a contaminated vehicle or a secondary transfer from a surface contaminated by the weapon. The other two had no explanation for that result in their questionnaire. However, all three shared the same occupation, vehicle locksmith, and worked in the same garage. Thus, working in contact with vehicles might be an alternative source for OGSR 
compounds. Another explanation might be a tertiary transfer from a contaminated colleague as the individuals worked in the same garage. Among the individuals for which only one compound was detected (18 on the hands and 12 on the sleeves), three had the same compound on their hands and sleeves (twice 4-nDPA and once AK II). None of them had contact with firearms. One was a consultant, the other a mechanic and the last one a student. Except for five one-compound positive specimens that might be explained by the possession of a firearm, the origin of the positive results is unknown. It is also worth mentioning that three individuals had occupations related to cars, adding to the three two-compounds positive results related to those professions. Regarding the compounds that were detected alone, AK II was the most frequent (11 and 7 positives for hands and sleeves respectively) followed by 4-nDPA (5 positives for both hands and sleeves).

In summary, the present results show that finding one compound on the hands of a civilian is not a rare occurrence, as the hands and sleeves of 18 and 12 individuals respectively tested positive for one compound (for a total of $24.6 \%$ of the studied civilian population, hands and sleeves combined). However, the presence of more than one OGSR compound remains unlikely (less than $7 \%$ of the studied population with two explanations related to firearms). Results also indicate that some activities in relation to vehicles might represent an alternative source for some OGSR compounds. However, this should be confirmed by further research.

\subsection{Police population}

For the prevalence study of the police population, 115 individuals from three Swiss police services were sampled. The population was composed of 97 men (84.3\%) and 18 women (15.7\%). All had a service weapon except for one scientific collaborator. Regarding the firearm brand and model, all individuals possessed a $9 \mathrm{~mm}$ Luger calibre service pistol from different brands (Fig. 4a). 30 individuals practiced shooting at least once a week (often: 26.1\%), 30 at least once a month (regularly: 26.1\%) and for 54 individuals at least once a year (rarely: 46.9\%) (Fig. 4b). The date of the last shooting session might also provide information as to the probability of a positive OGSR result and was reported in Figure 4c. Indeed, 23 individuals (20\%) had just finished a shooting session at the time of specimen collection. The amounts on their hands and sleeves were therefore expected to be much higher than for individuals who last practiced more than one week prior. 44 individuals had practiced shooting in the last 24 hours, six in the last eight days and for 40 , the last shooting training was more than eight days ago. As to the number of cartridges fired during training, 52 individuals (45.2\%) used between 51 and 100 cartridges and 45 (39.1\%) between 11 and 50 cartridges (Fig. 4d). Consequently, the level of contamination from a police officer after training might be very high. Lastly, the individuals were asked about shooting activities outside work. Most of the sampled police officers (94 people: $81.7 \%$ ) did not practice shooting as a hobby. 
a) Firearm model

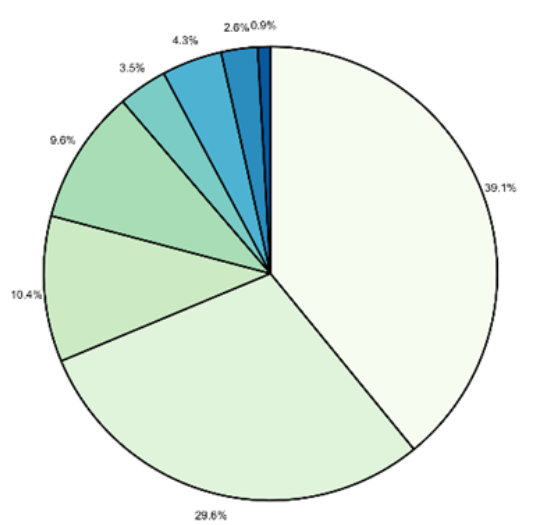

c) Last shooting training

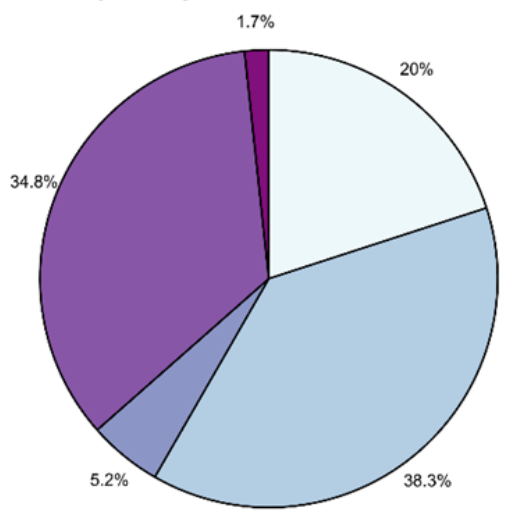

b) Firearm use frequency

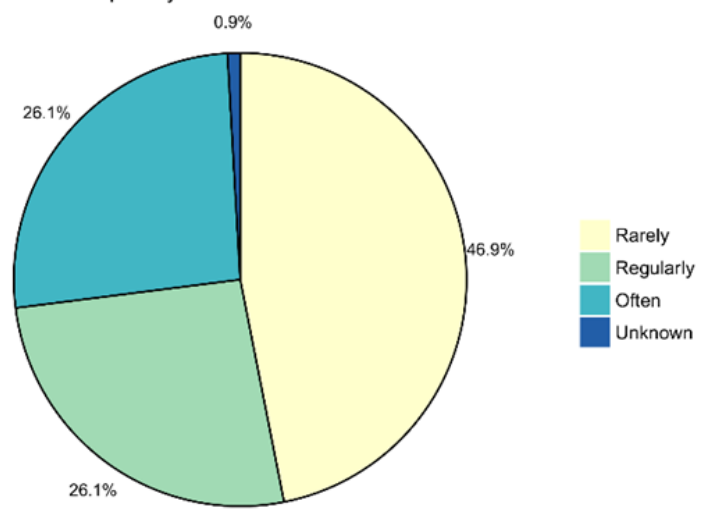

d) Number of cartridges discharged

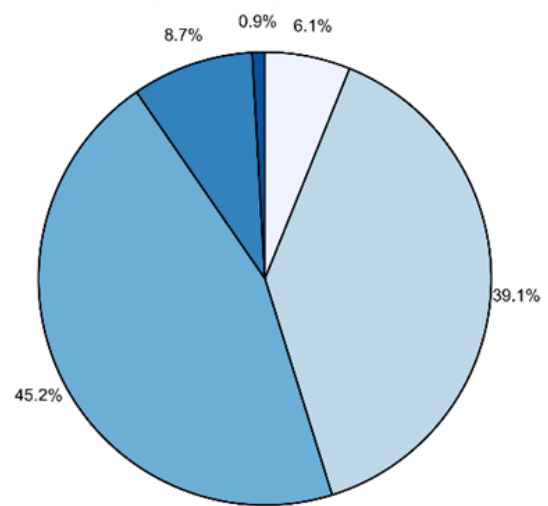

$1-10$

$11-50$

$51-100$

$>100$

Figure 4: Distribution of the police population in terms of a) Firearm model, b) Frequency of firearm use, c) Last shooting training and d) Number of cartridges discharged $(n=115)$

In the police population, the hands of $73(63.5 \%)$ and the sleeves of $77(67 \%)$ individuals tested negative for OGSR (Fig.5a). One compound was detected in about $10 \%$ of the individuals for both hands and sleeves. Two and three compounds were less frequently detected (about $5 \%$ ). Four or more compounds were found on the hands/sleeves of 19 and 18 individuals respectively ( $16.5 \%$ and $15.6 \%$ respectively). When adding the numbers of compounds on hands and sleeves, only 62 individuals (53.9\%) were free from OGSR. Specimens from 25.2\% (29 people) gave a total of four or more compounds. Interestingly, the police prevalence of one compound was slightly lower than that observed in the civilian population ( 9.6 vs $14.8 \%$ on hands).

Regarding the occurrence of each compound (Fig. 5b), $N$-nDPA was the most frequently encountered compound, with 34 occurrences on hands and 27 on sleeves. EC was the second most common compound, followed by DPA derivatives, AK II and DPA. Finally, $N, N$-DPF was more rarely detected, but this might also be due to its very low proportion in smokeless powders. MC was only detected once, indicating that it may not be present or only at a very low percentage in the ammunition used by the police forces. 


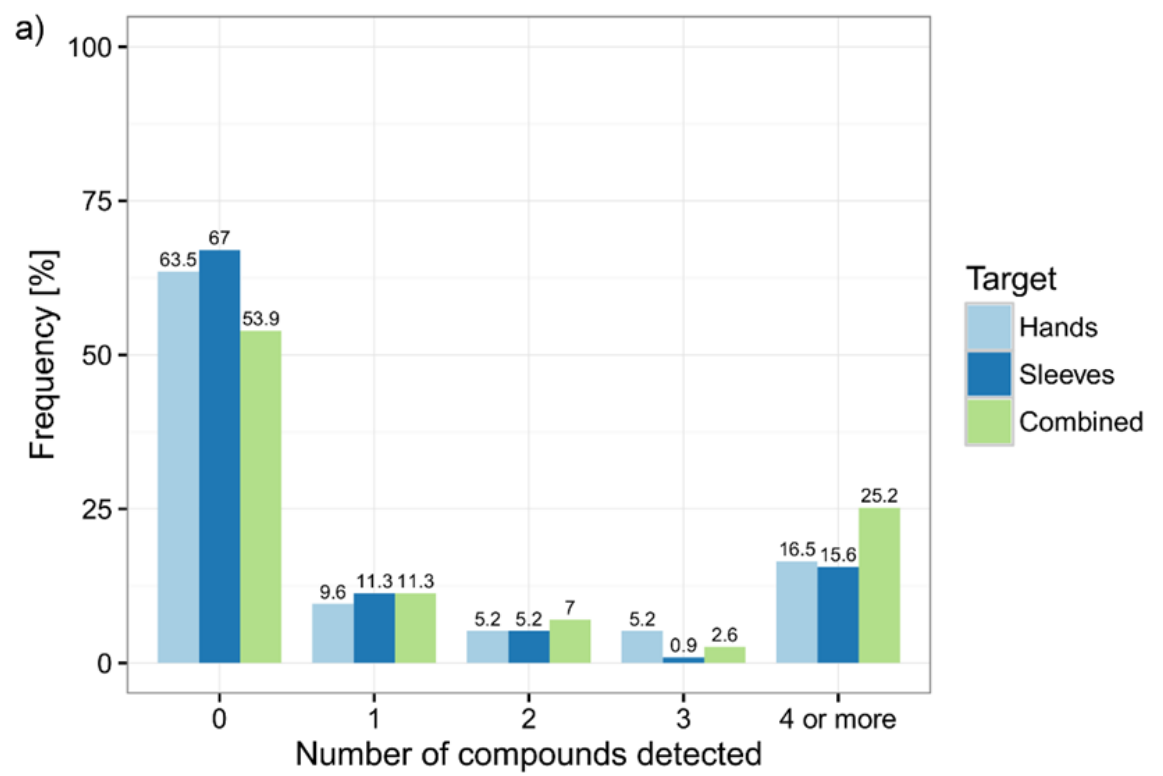

b)

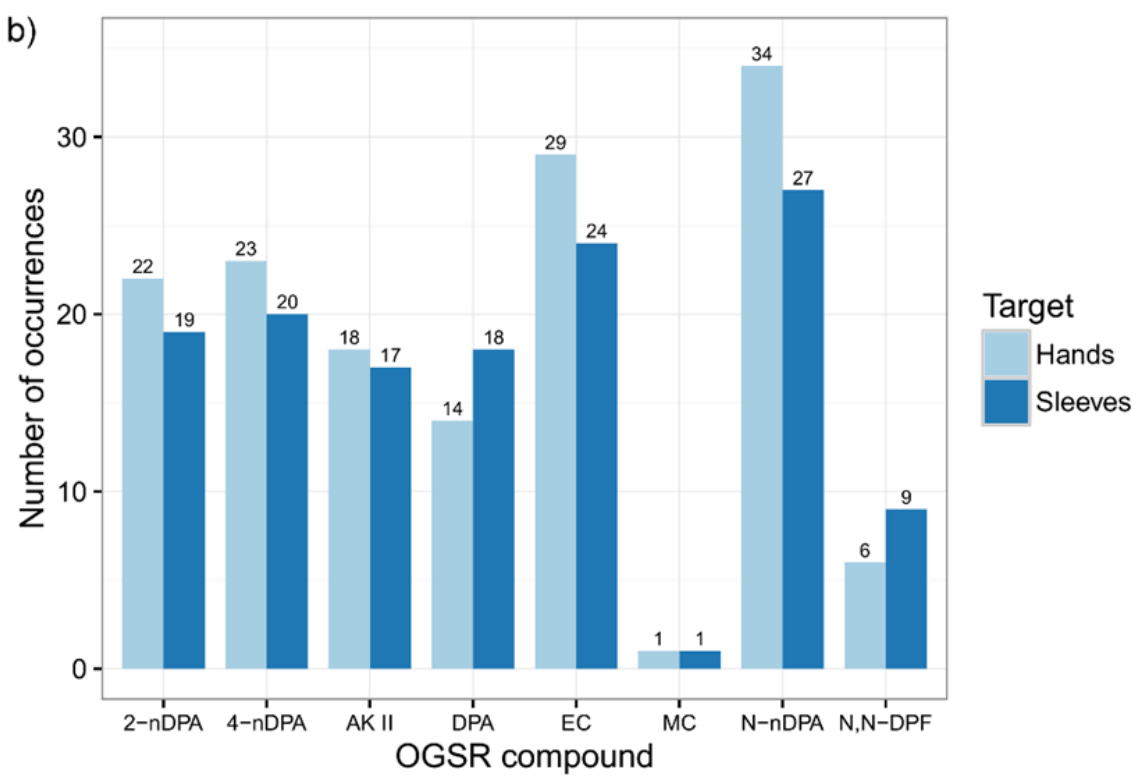

Figure 5: Prevalence in the police population. a) Prevalence versus number of compounds detected. The "combined" bar considers the total number of compounds detected on both hands and sleeves irrespective of potential compound duplication. For example, if DPA was detected on the hands and the same compound was detected on the sleeves, two compounds were counted. b) Number of positive specimens per analyte

There was a substantial variation in the amounts of OGSR compounds recovered, with values ranging from zero to values above the highest calibration standard (Fig. 6). The highest amounts were observed for DPA and $N$-nDPA. The other compounds were detected at tenfold lower concentrations. This might be explained by the ammunition used by police services, as the composition of smokeless powders has been shown to vary between brands [19]. Thus, DPA might be a major stabilizer in the smokeless powders used by the collaborators of the three police services sampled in this study. 


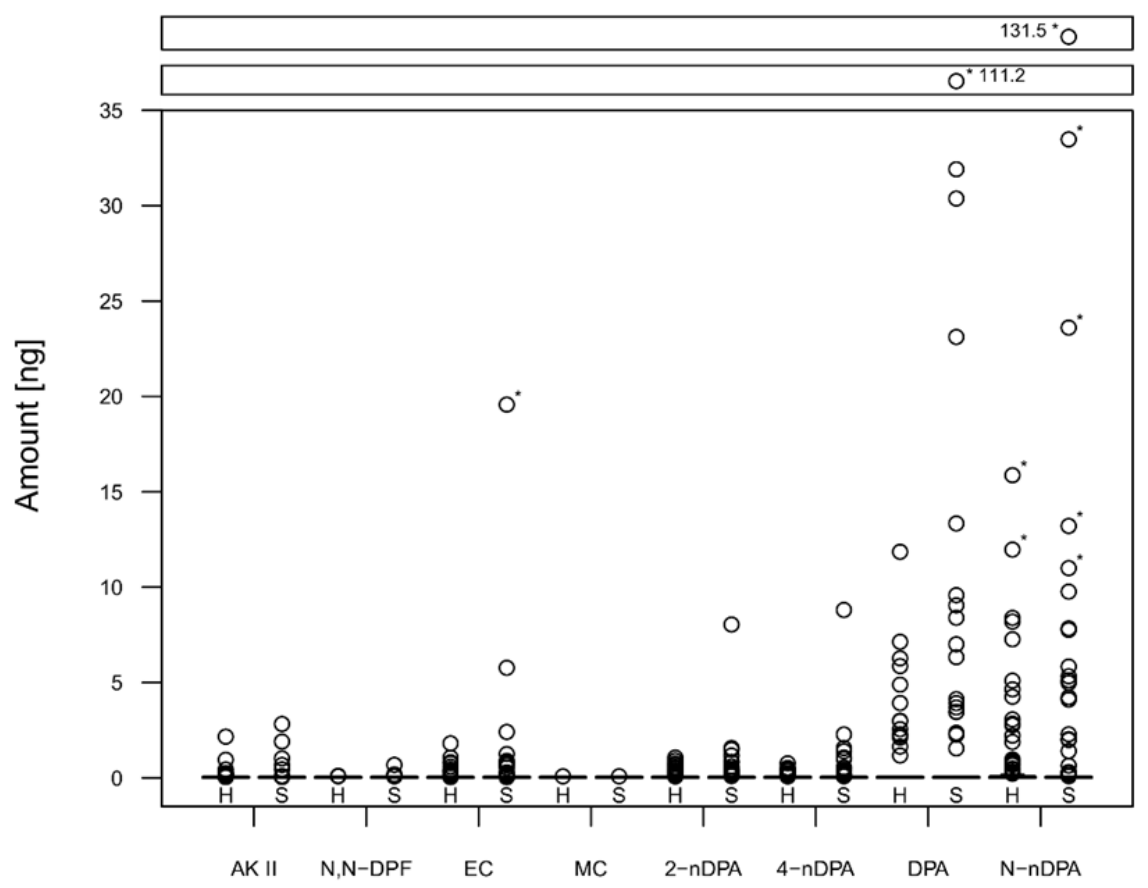

Figure 6: Prevalence in the police population: Amount of analyte detected. The asterisks indicate extrapolated values (outside of calibration range)

As expected, the highest number of compounds was detected on individuals who had just finished their shooting training $(<10 \mathrm{~min})$. All the specimens from their hands tested positive for OGSR and a total number of seven compounds were detected for four of them (Fig. 7a). Such a high number was never detected for individuals for whom the time since last shooting was over ten minutes. It is interesting to emphasize that in the less than $24 \mathrm{~h}$ category, two and three compounds were detected on the hands of individuals who had discharged a firearm less than three hours before. However, five and four compounds respectively were found on the hands of two people more than $24 \mathrm{~h}$ after shooting. A similar trend was observed for the wrists/sleeves specimens as the highest numbers of compounds (up to eight compounds) were generally detected from the sleeves of individuals who had just finished a shooting session (Fig. 7b). Four to six compounds were detected on the sleeves of four people who fired less than $24 \mathrm{~h}$ before. Interestingly, the two cases with six compounds and the one with four compounds were from individuals who practiced shooting less than three hours ago. After more than 24 hours, a maximum of one compound was detected on the sleeves.

As illustrated in Fig.7a-b, the distribution of the number of compounds varied with the elapsed time. Logically, the highest numbers of compounds were detected just after firing and due to losses related to various activities, lower numbers were detected afterwards. Thus, the detection of a high number of OGSR compounds can be related to a recent discharge. Persistence studies drew attention to the rapid decrease in OGSR quantities $[39,40]$. Therefore, a decrease in the number of detected compounds may be due to the early disappearance of less concentrated compounds. 
a)

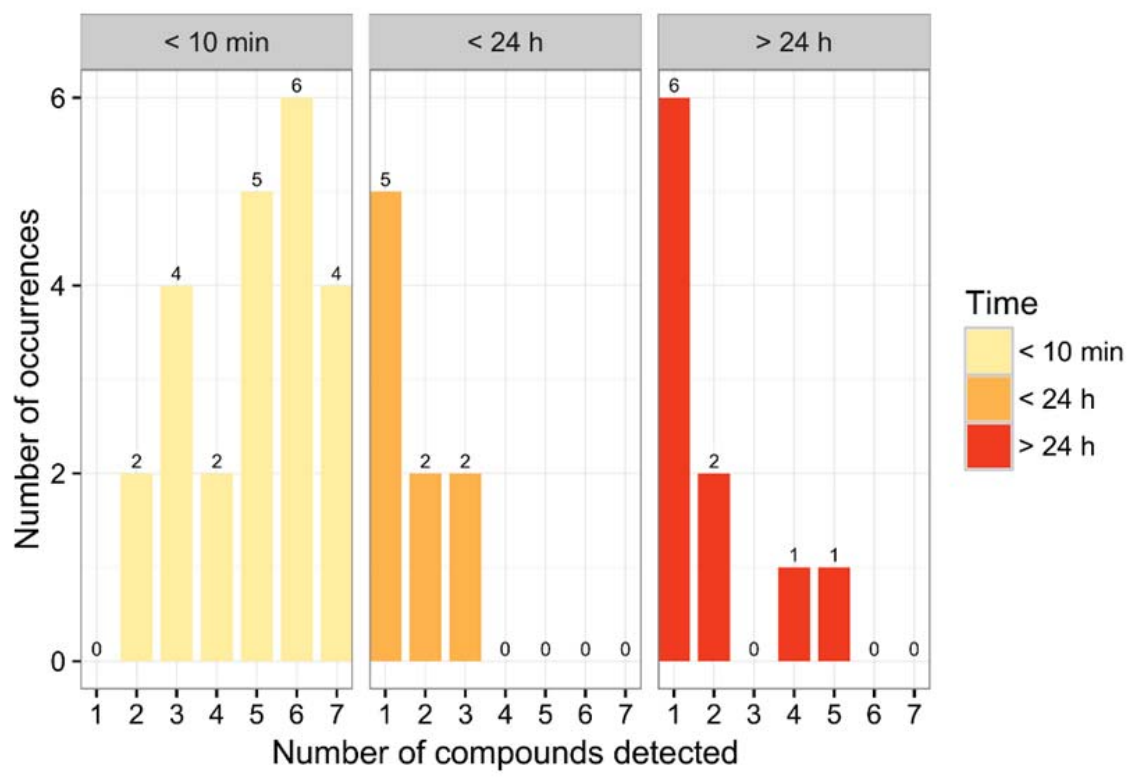

b)

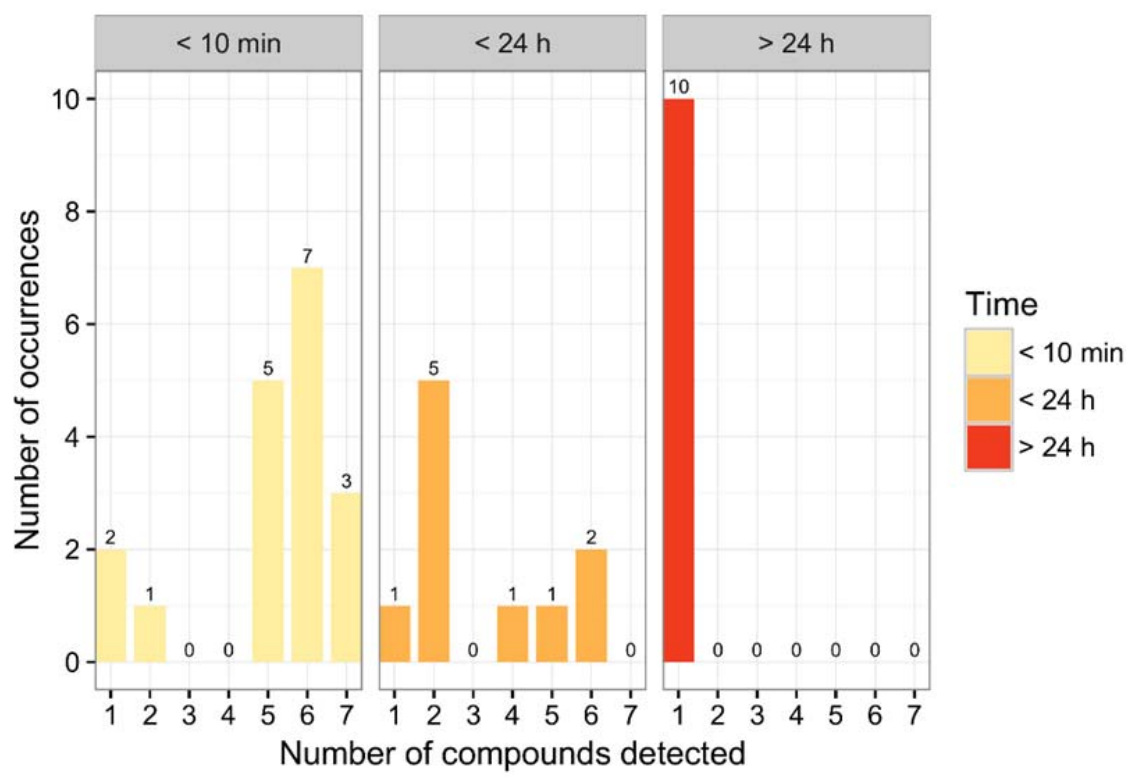

Figure 7: Number of OGSR compounds detected on a) police officers' hands, b) police officers' sleeves vs time elapsed

The detection of four or more compounds was generally related to individuals who had discharged a firearm in the last few hours before specimen collection. However, in two cases, four and five compounds were detected on the hand specimens of people who had not discharged a firearm recently ( $>24 \mathrm{~h}$ ). On referring to the questionnaire data, it was revealed that one of these two police officers had shaken hands with someone who had just left shooting training (five compounds) and the other had handled his service weapon not long before OGSR collection (four compounds). Weapon manipulation also explained the two instances with two compounds on the hands after $>24 \mathrm{~h}$. These results imply that it is rare to detect more than one OGSR compound beyond 24 hours without a 
secondary transfer explanation. Two recent studies highlighted secondary transfer during a handshake or firearm displacement [10,41]. For police officers, manipulation of a firearm involves generally more than ten seconds of contact, as investigated in the first aforementioned study [10]. Depending on the background contamination and the intensity of the manipulation, such as opening the breech or removing the charger, a high degree of secondary transfer might occur as confirmed in the present study and in the second aforementioned study [41]. For the positive specimens containing one compound, the results often remained unexplained, whether for hand or wrists specimens. It is also worth emphasizing that wrist/sleeve specimens collected from five individuals who had just finished training were negative. Thus, if the detection of OGSR compounds generally implies a firearm discharge, a negative result shortly after discharge is also possible. While the aforementioned observations demonstrate the contamination risks in a police environment, more research will be necessary to understand the different factors at play in the transfer and persistence of OGSR. Contact with contaminated colleagues, manipulation of weapons, contaminated equipment (e.g. clothing, handcuffs) and premises (e.g. tables, computer, cars) are potential sources of OGSR positive specimens. Moreover, while detecting four or more compounds seems highly correlated with a recent firearm discharge, the presence of one compound appears to be a usual occurrence in such environment.

\subsection{Discussion}

Among the 122 civilians sampled in this study, 22 (18\%) had one or more compounds on their hands and $14(11.5 \%)$ on their wrists or sleeves. Compared to other studies, such prevalence appears to be relatively high. Bell and Seitzinger obtained less than 5\% positive results for a sample of 73 people [35]. Two other studies by Northrop [19] and Hofstetter et al. [36] found no positives in population samples of 100 and 27 individuals respectively. Three factors might explain this difference:

- the targeted country

- the analytical technique

- the sampled population

Bell/Seitzinger and Northrop collected specimens in the United States, while both the present study and that from Hofstetter et al. were carried out in Switzerland. While Switzerland is a country where many citizens own a gun due to mandatory military service, shooting sport and hunting tradition, the United States is also known for a relatively high firearm possession. Indeed, the firearm density in Switzerland has been estimated to 27.6 firearms per 100 inhabitants (firearm possession in the present study was 22.1 ), whereas a value of 120.5 was given for the United States [1]. Thus, the targeted country is probably not the main influence factor for OGSR prevalence.

Three different analytical techniques were used to study prevalence in previous studies. Bell and Seitzinger used IMS, Northrop used MEKC and Hofstetter et al. used LC-MS for their respective studies. The sensitivity can vary considerably between analytical approaches. For example, IMS is the 
least sensitive, with reported LOD for DPA and EC of 50 and $1 \mathrm{ng}$ respectively [42], while using LCMS/MS, LOD of 0.5 and $0.01 \mathrm{ng}$ can be attained, respectively. Capillary electrophoresis is the most sensitive technique in terms of amounts of injected analyte with a LOD of $1.8 \mathrm{pg}$ and $0.9 \mathrm{pg}$ for EC and DPA, respectively [19]. However, the injection volume is also much lower (in the nanoliter range) than those used with LC-MS/MS instrumentation and LOD should be compared in terms of concentrations and not amounts loaded in the capillary or column. As the exact injection volume was not indicated in Northrop study, the better sensitivity of LC-MS vs CE can only be extrapolated. Thus, sensitivity issues might explain the differences observed between Bell and Seitzinger's study, Northrop's and the present study.

Finally, the sample size and selection can also have a considerable influence on the results. Indeed, the number of individuals involved in the studies remained very limited (from 27 to 122) and might not be representative of the whole population due to the absence of randomization in the participant selection. For example, in the present study 68 students were involved (55.7\% of the whole dataset), yielding a much younger population in comparison to the whole country. Consequently, larger prevalence studies would allow a more accurate picture of OGSR prevalence. It might also be interesting to target populations close to a person of interest in casework, as the prevalence might vary with the living area (city/countryside, known firearm violence, etc).

This study highlighted the possibility to detect several OGSR compounds on an individual without reported contact with firearms. Thus, while most positive results with three or more compounds could be explained by the questionnaires, one individual had four compounds on his sleeves without explanation (i.e. did not report any contact with firearms or potential secondary transfer). Given the other results from the study, such a high number of OGSR-related compounds supports the hypothesis of primary or secondary transfer rather than an alternative source. On the contrary, the presence of a single OGSR compound is more frequent and can derive from an alternative source. For example, DPA has multiple applications in the perfume industry and is used to prevent oxidation in rubber and scald of some fruit crops [43]. Goudsmits et al. compiled the results of a number of OGSR publications and listed the compounds appearing frequently in propellant formulations and having few alternative sources [31]. According to their study, EC, MC, nitroglycerin and nitroguanidine might be the most relevant OGSR targets. While our results confirm that EC and MC were very rarely detected in a civilian population, nitroglycerin and nitroguanidine were not targeted. The first can only be detected in negative mode, which was not used in this study, and the second is only found in triple base smokeless powders that are exclusively used in large calibre ammunition. Logically, the police population prevalence was noticeably higher, with $36.5 \%$ and $33 \%$ of the hand and wrist specimens testing positive for one or more compounds respectively. A previous study investigating the prevalence of OGSR for police officers found only two people positive to OGSR out of $25(7.4 \%)$ [36]. However, the population was composed of people working in a forensic laboratory and not police officers holding a service weapon and practicing shooting, which might explain the 
difference in prevalence. Contamination in the police environment is not limited to police officers, as Ali et al. also demonstrated the presence of OGSR in police stations and vehicles [37]. Our study also highlights that a vehicle locksmith who installed material in the trunk of a police vehicle had two OGSR compounds on his hands and on his sleeves, indicating a potential secondary transfer from a contaminated source. It would be interesting to collect more information regarding OGSR background level in police premises, where a suspect might be brought during investigation. Indeed, if there is a non-negligible background level, secondary transfer to people transported and/or detained in police premises might occur.

By comparing both populations, one can observe that OGSR prevalence is about twice as high for police officers ( $\geq$ one compound: $36.5 \%$ on hands and $33 \%$ on sleeves) than for civilians ( $\geq$ one compound: $18 \%$ on hands and $11.5 \%$ on sleeves). This is expected, as most civilians do not have regular contact with firearms. In the civilian population, the prevalence decreased as the number of compounds detected together increased. Only four individuals (less than 5\%) had two or more compounds on the hands and/or sleeves (if we exclude the person who discharged a firearm less than 10 minutes before specimen collection). In the police population, the trend was dependent on the time elapsed since discharge. Indeed, the highest numbers of compounds were in most cases observed for individuals who discharged a firearm very recently (or explained through secondary transfer). After 24 hours, the distribution in the police population was more similar to the civilian one. In terms of compound prevalence, the two most frequently detected compounds were AK II and 4-nDPA in the civilian population, whereas $N$-nDPA and EC were the most frequently detected in the police population. It is interesting to note that in the police population, most of the one compound positive results (hands and sleeves considered together) concerned AK II, potentially indicating a longer persistence for that particular compound. On the contrary, MC was very rarely detected in both populations. As discussed above, finding traces of one OGSR compound on the hands of an individual can occur without an explanation. Indeed, alternative sources were previously reported for some OGSR compounds, such as DPA. In this case, how relevant would be the detection of DPA alone? As highlighted by others $[10,18]$, the simultaneous detection of several compounds has greater value than that of a single compound. This was also confirmed in the present study, as the detection of more than three OGSR compounds together was generally related to a direct or indirect contact with firearms. The only exception was a civilian with four compounds on his sleeves. However, while there was no explanation as to the exact source of this result, its origin is more likely to be from a firearm than from an environmental source. In practice, it would be important to investigate the risk of contamination through secondary transfer. One should keep in mind that the number of OGSR compounds that is detected simultaneously also depends on the combination firearm-ammunition, that is to say the number of compounds present in the propellant and a potential memory effect. Hence, the case-bycase approach proposed by Romolo and Margot recommending to evaluate evidence in light of case circumstances and using whenever possible comparison of GSR with other case-specific items such as 
the cartridge case is particularly attractive [44]. Finally, some of the civilians positive to OGSR and without contact with firearms were vehicle locksmiths. It would then be advisable to look for alternative sources in the automotive industry. It must be noted that vehicle parts, more particularly brake pads [45-47], were also mentioned as potential alternative sources for IGSR, though a recent study indicated that it is not a concern anymore [48].

\section{CONCLUSIONS}

The present work investigated the prevalence of eight OGSR compounds in a civilian and police population to provide information for the assessment of that trace. Specimens were collected on the hands and sleeves of 122 civilians and 115 individuals working in a police environment using carbon stubs. One stub was used to sample both hands and the other was used to sample the cuffs of the sleeves or the wrists when a short-sleeve upper garment was worn. Data was acquired using liquid chromatography coupled to tandem mass spectrometry (LC-MS/MS) with electrospray ionisation in positive mode.

Prevalence in the civilian sample was relatively high, with $18 \%$ of the 122 civilians sampled having one or more compounds on their hands and $11.5 \%$ on their wrists or sleeves. More than three compounds were detected simultaneously from only two individuals. Using data from the questionnaire, it was possible to explain one result by a direct contact with firearms. However, no explanation was found in the questionnaire for the presence of four compounds on the sleeves of the second person. Results also showed that secondary transfer (through handshake or service weapon handling) can explain OGSR contamination. The detection of two compounds on three vehicle locksmiths might indicate alternative sources (possibly related to occupations in the automotive field). Further research is required to investigate such alternative sources.

For the police population, the prevalence was higher, with $36.5 \%$ of the hand specimens and $33 \%$ of the wrist specimens positive to one or more compounds. A higher prevalence was expected for this population due to service weapon holding and regular shooting exercises. In the civilian population, the prevalence decreased as the number of compound detected simultaneously increased, whereas in the police population, the trend was dependent on the time elapsed since the last shooting exercise. Indeed, the simultaneous detection of four or more compounds was in most cases observed for individuals who discharged a firearm very recently $(<10$ minutes). Due to the relatively high prevalence of OGSR on police officers, it would be interesting to target their premises (police stations and vehicles for example) to evaluate the background contamination. This is critical, as an arrested person might be contaminated by secondary transfer from a contaminated vehicle, a police officer or even a desk at a police station. In any case, it would serve as a quality assurance tool for laboratory contamination monitoring if OGSR is implemented in routine work in parallel to IGSR analysis. From the present results, it appears that there is an important prevalence for single compounds. The 
518 simultaneous detection of two compounds shows a lower prevalence and the detection of three or

519 more compounds supports the hypothesis of a firearm origin rather than an environmental source.

520 However, results should be interpreted on a case-by-case basis, as the number of compounds that can

521 be detected depends on the composition of the propellant.

522 Further prevalence studies on a larger scale should be carried out to avoid bias in participant selection

523 and provide a more representative picture of the population. The present study was performed in ESI

524 positive mode and detecting additional compounds in the negative mode, such as dinitrotoluenes and

525 nitroglycerine, would be valuable. Further research for OGSR environmental sources is also required

526 and should be combined with prevalence data to establish the most relevant compounds and evaluate

527 what combinations of compounds are the most characteristic for firearm discharge. Such information

528 would be particularly useful for OGSR interpretation in casework.

529

530 Acknowledgements

531

532 The authors wish to acknowledge the Swiss National Science Foundation (10521A_165608) for

533 financial support. They also would like to thank three Swiss police services for enabling the collection

534 of the police prevalence specimens, especially Commissaires Nicola Albertini and Daniel Kolly from

535 the Police Cantonale Vaudoise, Oberleutnant Adrien Cordier and Adjutant Dr Balthasar Jung from the

536 Kantonspolizei Aargau and Stephan Christen from the Forensisches Institut Zürich. They are grateful

537 to Dr Amanda Frick for proof reading this manuscript. Finally, they would like to warmly thank all the

538 volunteers who took part to the study.

539 
542 [1] Karp A. Estimating Global Civilian-Held Firearms Numbers. Small Arms Survey; 2018. p. 1-12.

543 [2] Zeichner A. Firearm Discharge Residue: Analysis of. In: John Wiley \& Sons L, editor. Wiley 544 Encyclopedia of Forensic Science2009.

545 [3] Meng HH, Caddy B. Gunshot residue analysis - A review. J Forensic Sci. 1997;42:553-70.

546 [4] Zeichner A. Recent developments in methods of chemical analysis in investigations of firearmrelated events. Anal Bioanal Chem. 2003;376:1178-91.

[5] Romolo FS, Stamouli A, Romeo M, Cook M, Orsenigo S, Donghi M. An experimental study about the presence of selenium in inorganic gunshot residues (GSR). Forensic Chem. 2017;4:51-60.

[6] Charles S, Geusens N. A study of the potential risk of gunshot residue transfer from special units of the police to arrested suspects. Forensic Sci Int. 2012;216:78-81.

[7] French J, Morgan R. An experimental investigation of the indirect transfer and deposition of gunshot residue: further studies carried out with SEM-EDX analysis. Forensic Sci Int. 2015;247:14-7.

[8] French J, Morgan R, Davy J. The secondary transfer of gunshot residue: an experimental investigation carried out with SEM-EDX analysis. X-Ray Spectrom. 2014;43:56-61.

[9] Brozek-Mucha Z. On the prevalence of gunshot residue in selected populations - An empirical study performed with SEM-EDX analysis. Forensic Sci Int. 2014;237:46-52. [10] Gassner AL, Manganelli M, Werner D, Rhumorbarbe D, Maitre M, Beavis A, et al. Secondary transfer of organic gunshot residues: Empirical data to assist the evaluation of three scenarios. Sci Justice. 2019;59:58-66. gunshot residue particles in the random population. Forensic Sci Int. 2016;262:150-5.

[12] Gialamas DM, Rhodes EF, Sugarman LA. Officers, Their Weapons and Their Hands - an EmpiricalStudy of Gsr on the Hands of Non-Shooting Police Officers. J Forensic Sci. 1995;40:1086-9.

[13] Gerard RV, Lindsay E, McVicar MJ, Randall ED, Gapinska A. Observations of Gunshot Residue Associated with Police Officers, Their Equipment, and Their Vehicles. Canadian Society of Forensic Science Journal. 2012;45:57-63.

[14] Cook M. Gunshot residue contamination of the hands of police officers following start-of-shift handling of their firearm. Forensic Sci Int. 2016;269:56-62.

[15] Lindsay E, McVicar MJ, Robert V. Gerard RV, Randall ED. Observations of GSR on the Hands of Employees at Firearms Manufacturing Facilities. Canadian Society of Forensic Science Journal. 2011;44:105-9.

[16] Berk RE, Rochowicz SA, Wong M, Kopina MA. Gunshot residue in Chicago police vehicles and facilities: An empirical study. J Forensic Sci. 2007;52:838-41.

[17] Hannigan TJ, McDermott SD, Greaney CM, O'Shaughnessy J, O'Brien CM. Evaluation of gunshot residue (GSR) evidence: Surveys of prevalence of GSR on clothing and frequency of residue types. Forensic Sci Int. 2015;257:177-81.

[18] Maitre M, Kirkbride KP, Horder M, Roux C, Beavis A. Current perspectives in the interpretation of gunshot residues in forensic science: A review. Forensic Sci Int. 2017;270:1-11.

[19] Northrop DM. Gunshot residue analysis by micellar electrokinetic capillary electrophoresis: Assessment for application to casework. Part I. J Forensic Sci. 2001;46:549-59.

[20] Northrop DM. Gunshot residue analysis by micellar electrokinetic capillary electrophoresis: Assessment for application to casework. Part II. J Forensic Sci. 2001;46:560-72.

[21] Reardon MR, MacCrehan WA, Rowe WF. Comparing the additive composition of smokeless gunpowder and its handgun-fired residues. J Forensic Sci. 2000;45:1232-8.

[22] MacCrehan WA, Patierno ER, Duewer DL, Reardon MR. Investigating the effect of changing ammunition on the composition of organic additives in gunshot residue (OGSR). J Forensic Sci. 2001;46:57-62.

[23] Muller D, Levy A, Vinokurov A, Ravreby M, Shelef R, Wolf E, et al. A novel method for the analysis of discharged smokeless powder residues. J Forensic Sci. 2007;52:75-8. 
[24] Zeichner A, Eldar B. A novel method for extraction and analysis of gunpowder residues on double-side adhesive coated stubs. J Forensic Sci. 2004;49:1194-206.

593 [25] Tarifa A, Almirall JR. Fast detection and characterization of organic and inorganic gunshot residues on the hands of suspects by CMV-GC-MS and LIBS. Sci Justice. 2015;55:168-75.

595 [26] Laza D, Nys B, De Kinder J, Mesmaeker AKD, Moucheron C. Development of a quantitative LC$596 \mathrm{MS} / \mathrm{MS}$ method for the analysis of common propellant powder stabilizers in gunshot residue. J 597 Forensic Sci. 2007;52:842-50.

598 [27] Thomas JL, Lincoln D, McCord BR. Separation and Detection of Smokeless Powder Additives by Ultra Performance Liquid Chromatography with Tandem Mass Spectrometry (UPLC/MS/MS). J Forensic Sci. 2013;58:609-15.

[28] Benito S, Abrego Z, Sanchez A, Unceta N, Goicolea MA, Barrio RJ. Characterization of organic gunshot residues in lead-free ammunition using a new sample collection device for liquid chromatography-quadrupole time-of-flight mass spectrometry. Forensic Sci Int. 2015;246:79-85.

[29] Taudte RV, Roux C, Blanes L, Horder M, Kirkbride KP, Beavis A. The development and comparison of collection techniques for inorganic and organic gunshot residues. Anal Bioanal Chem. 2016;408:2567-76.

[30] Gassner AL, Weyermann C. LC-MS method development and comparison of sampling materials for the analysis of organic gunshot residues. Forensic Sci Int. 2016;264:47-55.

[31] Goudsmits E, Sharples GP, Birkett JW. Preliminary classification of characteristic organic gunshot residue compounds. Sci Justice. 2016;56:421-5.

[32] Crowson CA, Cullum HE, Hiley RW, Lowe AM. A survey of high explosives traces in public places. J Forensic Sci. 1996;41:980-9.

[33] Cullum HE, McGavigan C, Uttley CZ, Stroud MAM, Warren DC. A second survey of high explosives traces in public places. J Forensic Sci. 2004;49:684-90.

[34] Lahoda KG, Collin OL, Mathis JA, LeClair HE, Wise SH, McCord BR. A survey of background levels of explosives and related compounds in the environment. J Forensic Sci. 2008;53:802-6.

[35] Bell S, Seitzinger L. From binary presumptive assays to probabilistic assessments: Differentiation of shooters from non-shooters using IMS, OGSR, neural networks, and likelihood ratios. Forensic Sci Int. 2016;263:176-85.

[36] Hofstetter C, Maitre M, Beavis A, Roux CP, Weyermann C, Gassner AL. A study of transfer and prevalence of organic gunshot residues. Forensic Sci Int. 2017;277:241-51.

[37] Ali L, Brown K, Castellano H, Wetzel SJ. A Study of the Presence of Gunshot Residue in Pittsburgh Police Stations using SEM/EDS and LC-MS/MS. J Forensic Sci. 2016;61:928-38.

[38] Zeichner A, Levin N. Collection Efficiency of Gunshot Residue (Gsr) Particles from Hair and Hands Using Double-Side Adhesive Tape. J Forensic Sci. 1993;38:571-84.

[39] Gassner AL, Ribeiro C, Kobylinska J, Zeichner A, Weyermann C. Organic gunshot residues: Observations about sampling and transfer mechanisms. Forensic Sci Int. 2016;266:369-78.

[40] Maitre M, Horder M, Kirkbride KP, Gassner AL, Weyermann C, Roux C, et al. A forensic investigation on the persistence of organic gunshot residues. Forensic Sci Int. 2018;292:1-10.

[41] Maitre M, Chadwick S, Kirkbride PK, Gassner AL, Weyermann C, Beavis A, et al. An investigation on the secondary transfer of organic gunshot residues. Sci Justice. 2019;In Press.

[42] Yeager B, Bustin K, Stewart J, Dross R, Bell S. Evaluation and validation of ion mobility spectrometry for presumptive testing targeting the organic constituents of firearms discharge residue. Anal Methods-Uk. 2015;7:9683-91.

[43] Drzyzga O. Diphenylamine and derivatives in the environment: a review. Chemosphere. 2003;53:809-18.

[44] Romolo FS, Margot P. Identification of gunshot residue: a critical review. Forensic Sci Int. 2001;119:195-211.

[45] Cardinetti B, Ciampini C, D'Onofrio C, Orlando G, Gravina L, Ferrari F, et al. X-ray mapping technique: a preliminary study in discriminating gunshot residue particles from aggregates of environmental occupational origin. Forensic Sci Int. 2004;143:27-46. 
642 [46] Garofano L, Capra M, Ferrari F, Bizzaro GP, Di Tullio D, Dell'Olio M, et al. Gunshot residue 643 Further studies on particles of environmental and occupational origin. Forensic Sci Int. 1999;103:164421.

645 [47] Torre C, Mattutino G, Vasino V, Robino C. Brake linings: A source of Non-GSR particles containing 646 lead, barium, and antimony. J Forensic Sci. 2002;47:494-504.

647 [48] Tucker W, Lucas N, Seyfang KE, Kirkbride KP, Popelka-Filcoff RS. Gunshot residue and brakepads:

648 Compositional and morphological considerations for forensic casework. Forensic Sci Int. 649 2017;270:76-82.

650 